\title{
Human Platelet Lysate as Validated Replacement for Animal Serum to Assess Chemosensitivity
}

\author{
Miriam Pons 1, Georg Nagel1, Yanira Zeyn', Mandy Beyer1, Teresa Laguna², Tina Brachetti', Andreas Sellmer ${ }^{3}$, \\ Siavosh Mahboobi ${ }^{3}$, Roland Conradi4, Falk Butter² and Oliver H. Krämer' \\ ${ }^{1}$ Department of Toxicology, University Medical Center, Mainz, Germany; ${ }^{2}$ Institute of Molecular Biology, Mainz, Germany; ${ }^{3}$ Institute of Pharmacy, \\ Department of Pharmaceutical/Medicinal Chemistry I, University of Regensburg, Regensburg, Germany; ${ }^{4}$ Transfusion Centre, University Medical Center, \\ Mainz, Germany
}

\begin{abstract}
Experiments with cultured mammalian cells represent an in vitro alternative to animal experiments. Fetal calf serum (FCS) is the most commonly used medium supplement worldwide. FCS contains a variable mixture of growth factors and cytokines that support cell proliferation. This undefined nature of FCS is a source of experimental variation, undesired immune responses, possible contaminations and, because of the way it is obtained, an ethical concern. Thus, alternative, defined, valid, and reliable medium supplements should be characterized in a large number of experiments. Human platelet lysate (hPL) is increasingly appreciated as an alternative to FCS. Since it is unclear whether cells respond differentially to clinically relevant chemotherapeutics inducing replicative stress and DNA damage (hydroxyurea, irinotecan), induction of reactive oxygen species (ROS), the tyrosine kinase inhibitor (TKi) imatinib, and novel epigenetic modifiers belonging to the group of histone deacetylase inhibitors (HDACi), we investigated these issues. Here we show that cancer cells derived from leukemia and colon cancer grow very similarly in culture media supplemented with FCS or outdated hPL. Notably, cells have practically identical proteomes under both culture conditions. Moreover, cells grown with FCS or hPL responded equally to all types of drugs and stress conditions that we tested. In addition, the transfection of blood cells by electroporation can be achieved under both conditions. Furthermore, we reveal that class I HDACs, but not HDAC6, are required for the expression of the pan-leukemic marker WT1 under various culture conditions. Hence, hPL is a moderately priced substitute for FCS in various experimental settings.
\end{abstract}

\section{Introduction}

Cell culture experiments can replace a large number of animal experiments. However, FCS, a very frequently used additive in cell culture experiments (Gstraunthaler, 2003; Gstraunthaler et al., 2014), is drawn from unborn calves older than three months gestation and its collection represents a massive burden for the animals. A needle is inserted into the heart of the fetus to obtain blood and, since the animals are not under anesthesia, they may suffer pain and discomfort. Additional problems arising from the use of FCS are its potential to be a source of contaminations and the commonly observed variations in its quality, which make FCS an unreliable growth stimulator (Gstraunthaler, 2003; Gstraunthaler et al., 2014). Moreover, the addition of bovine proteins to human or mouse cell cultures, which are the cell types most frequently used in biomedical research, can lead to artifacts. For example, FCS can blur the identity of freshly isolated pancreatic cancer cells by its ability to excessively trigger otherwise not engaged signaling pathways (Moore et al., 2001). Moreover, the use of FCS is incompatible with donations of cells to humans for therapeutic purposes (Barsotti et al., 2013; Doucet et al., 2005). Due to these concerns, alternative strategies to the use of FCS have been considered.

While fully recombinant serum replacements are still a cost issue, human platelet lysate (hPL) could be a valid and ethically justifiable option. Human keratinocyte cells, renal epithelial cells, and various leukemic and solid tumor cell lines can be propagated in hPL (Fazzina et al., 2016; Rauch et al., 2011; Baik et al., 2014; Bernardi et al., 2013). This also holds true for primary human and rodent cells (adipocytes, amniotic fluid stem cells, bone marrow stromal cells, chondrocytes, corneal cells, endothelial cells, keratinocytes, mesenchymal stem cells, monocytes, osteoblasts) (Barsotti et al., 2013; Doucet et al., 2005; Glovinski et al., 2017). These cell types maintain functionality and signaling in various assays, and some reports even show a better cell proliferation in hPL than in FCS (Bernardi et al., 2013; Glovinski et al.,
Received September 21, 2018; Accepted November 23, 2018; Epub December 14, 2018; ( ) The Authors, 2018.

ALTEX 36(2), 277-288. doi:10.14573/altex.1809211

Correspondence: Oliver H. Krämer, PhD, Department of Toxicology, University Medical Center, Obere Zahlbacher Str. 67, 55131 Mainz, Germany (okraemer@uni-mainz.de)
This is an Open Access article distributed under the terms of the Creative Commons Attribution 4.0 International license (http://creativecommons.org/licenses/by/4.0/), which permits unrestricted use, distribution and reproduction in any medium, provided the original work is appropriately cited. 
2017; Barsotti et al., 2013). Assessment of DNA methylation patterns and the transcriptomes of mesenchymal stromal cells grown in hPL or FCS revealed very similar gene expression patterns under these conditions (Fernandez-Rebollo et al., 2017).

Furthermore, cells grown in xenogeneic-free hPL are therapeutically useful (Sergeeva et al., 2016; Riis et al., 2016; Thieme et al., 2017). They could be used for bone regeneration, osteoarthritis, the therapy of stroke, wound healing, and other applications (Tan et al., 2016; Sergeeva et al., 2016; Altaie et al., 2016; Martinelli et al., 2016; Riis et al., 2016; Lykov et al., 2017; Thieme et al., 2017; Leijs et al., 2017). Indeed, bone marrow stromal cells cultured in hPL are currently being tested in a phase I study involving patients with acute ischemic stroke (Shichinohe et al., 2017). Furthermore, primary cells grown in hPL maintain their genomic integrity and do not form tumors in vivo, a key requisite for clinical applications (Tan et al., 2016; Altaie et al., 2016; Martinelli et al., 2016).

Since fresh $\mathrm{hPL}$ is required for clinical use, it has been investigated whether outdated hPL could be a valid additive to cultivate adipose stem cells. Promisingly, outdated hPL is useful for this purpose (Rauch et al., 2011; Glovinski et al., 2017) and microarray analyses revealed no significant differences in gene expression profiles of cells cultured in fresh or outdated hPL (Glovinski et al., 2017).

As DNA-damaging chemotherapy is a mainstay of tumor therapy, the question was raised whether cells grown in hPL respond to such agents. The reactions of leukemic cells (KG-1a, HL60, Jurkat) to the DNA-damaging agent adriamycin in the presence of hPL or FCS are equal (Fazzina et al., 2016). However, it has not been clarified whether FCS and hPL differentially affect the DNA damage response that is triggered by a more diverse array of stimuli and in various cell types.

We assessed whether the reactions of leukemic cells to imatinib, hydroxyurea, and HDACi are affected variably by hPL and FCS. We carried out the same tests with colon cancer cells that we treated with irinotecan or the ROS inducer t-BOOH. Irinotecan inhibits topoisomerase- 1 and is a standard drug for colon cancer (Tomicic and Kaina, 2013; Rauch et al., 2018). We chose these agents based on their clinical relevance and on the fact that inducers of ROS and inhibitors of HDACs occur endogenously (Krämer, 2009; Newman and Verdin, 2014) and may hence be differentially contained in FCS and hPL. Hydroxyurea inhibits ribonucleotide reductase and can be used to reduce leukemic cell counts (Eklund et al., 2001; Nazha and Gerds, 2016), imatinib is used to treat BCR-ABL-positive chronic myeloid leukemia (CML) (Lamontanara et al., 2013). HDACi are more and more frequently used for leukemia patients and these agents are being considered for the therapy of further forms of cancer (Ceccacci and Minucci, 2016; Krämer et al., 2014). To assess cellular responses at the molecular level, we assessed how hydroxyurea, imatinib, and HDACi affect cancer-relevant molecules. These include the transcription factor Wilms tumor-1 (WT1), which is a marker for rapidly proliferating cells and cancer stem cells (Svensson et al., 2007; Makki et al., 2008; Ruan et al., 2018; Pons et al., 2018), and p53, which is seen as the guardian of the genome (Hofseth et al., 2004).

We show that leukemic cells and colon cancer cells grow in hPL equally well to slightly better than in FCS. Proteomics followed by mass spectrometry demonstrates in a previously not covered depth that the proteomes of leukemic cells cultured in hPL or FCS are practically identical. Moreover, both cell types responded equally to all stress conditions that we tested and the growth in hPL renders even hard-to-transfect hematopoietic cells suitable transfection hosts for siRNAs.

The use of hPL follows the principles of the 3Rs (Replacement, Reduction, Refinement) in order to provide alternatives to animal experiments. Our data suggest that hPL represents a reliable alternative for FCS in cell culture experiments. This could reduce or even replace FCS and thereby the collection of blood from unborn fetuses.

\section{Materials and methods}

\section{Generation of $h P L$}

Outdated human platelet concentrates in human plasma (2-4 $\mathrm{x}$ $10^{11} /$ unit; unit $=200-250 \mathrm{ml}$ ) were the source of hPL. They were received from healthy donors at the Transfusion Centre of the University Medical Center Mainz with sterile instruments. Samples were tested negative for hepatitis B, C and E, HIV, CMV, parvovirus B19, and Treponema pallidum. The concentrates had been manufactured by apheresis (aPC), and were no more than 3 days too old to be given to patients. Manufacturing and specifications of the aPC were in accordance with the German law (BMJV, 2005) and German guidelines (Bundesärztekammer, 2017). The concentrates were stored at $-80^{\circ} \mathrm{C}$. We worked with four independent pools of hPL generated by combining at least 3 platelet units each from a different donor. This strategy was chosen to reduce donor-dependent variations. Platelets were disrupted by freezing $\left(-80^{\circ} \mathrm{C}\right)$ and thawing $\left(37^{\circ} \mathrm{C}\right)$ cycles to generate platelet lysate. The lysates were centrifuged twice at $2600 \mathrm{xg}$ for $20 \mathrm{~min}$ to remove larger cell fragments, then sterile-filtered through a $0.22 \mu \mathrm{M}$ membrane.

\section{Cell lines and adaptation process}

We adapted three human leukemia cell lines (K562, NB4, and MV4-11) as well as HCT116 colon cancer cells to culture media (RPMI for K562, NB4, and MV4-11 and DMEM for HCT116 (media from Sigma-Aldrich)) containing 10\% FCS, 5\% FCS, $1 \%$ FCS (Gibco), $5 \%$ hPL or $1 \%$ hPL. To block coagulation, $2 \mathrm{U} / \mathrm{ml}$ heparin were added to the media containing hPL for K562, NB4 and MV4-11 cells and $4 \mathrm{U} / \mathrm{ml}$ were added for HCT116 cells. All media contained 1\% penicillin/streptomycin (Gibco); RPMI medium was additionally supplemented with $2 \%$ L-glutamine. Cells were adapted for at least 3 weeks before the first experiments. Every 3-4 days, cells were suspended firmly, passaged in a ratio of 1:15-1:20, and fresh medium was added. Adherent HCT116 cells were washed with phosphate buffered saline (PBS), detached from the culture flasks by trypsin/EDTA $(0.5 \mathrm{~g} / 1)$, and also passaged in a ratio of 1:15-1:20. We analyzed cell morphology by light microscopy (Axiovert 35, Carl Zeiss). Growth kinetics were examined by counting. Cell cycle distributions were investigated by flow cytometry (FACS Canto II, BD Biosciences). To assess whether cells grown with hPL can be frozen and thawed for continuous propagation, we froze cells in culture media containing $5 \% \mathrm{hPL}$ and $10 \% \mathrm{DMSO}$ at $-80^{\circ} \mathrm{C}$ overnight and then in liquid nitrogen for up to about one year. Thawed cells grew similarly to 
those that had been frozen with $10 \%$ FCS. All cells were in culture for no longer than three months and were tested monthly for mycoplasma contamination. K562 and NB4 cells were provided by Dr Manuel Grez (Frankfurt, Germany), MV4-11 cells by Prof. Dr Frank-Dietmar Böhmer (Jena, Germany), and HCT116 cells by Prof. Dr. Bert Vogelstein (Maryland, USA). Moreover, cells were authenticated by DNA fingerprint as specified (Pons et al., 2018; Göder et al., 2018).

\section{Drugs and chemicals}

Romidepsin (stock solution: $1 \mathrm{mM}$ in DMSO), entinostat (stock solution: $5 \mathrm{mM}$ in DMSO), and irinotecan (stock solution: $10 \mathrm{mM}$ in DMSO) were from Selleckchem; hydroxyurea (stock solution: $100 \mathrm{mM}$ in PBS) was from Sigma-Aldrich; and imatinib (stock solution: $1 \mathrm{mM}$ in DMSO) and marbostat-100 (stock solution: $10 \mathrm{mM}$ in DMSO) were synthesized by A. Sellmer and S. Mahboobi. Marbostat-100 is patented as Mahboobi, Siavosh et al., Preperation of fused heterocyclic compunds as HDAC6 inhibitors and their uses; WO2016020369A1. Except for hydroxyurea, which was always freshly prepared right before treatment, all stock solutions were stored at $-80^{\circ} \mathrm{C}$. All drugs were diluted in PBS before treatment. Purity of all drugs from Selleckchem and Sigma-Aldrich was $\geq 98 \%$ (HPLC). Imatinib and Marbostat- 100 also had purities of at least 98\% (imatinib: ${ }^{1} \mathrm{H}-\mathrm{NMR}$; marbostat-100: ${ }^{1} \mathrm{H}-\mathrm{NMR}$ and elemental analysis).

\section{Western blot}

Antibodies were from Santa Cruz Biotechnology: p53 (sc-81168); Cell signaling: E-Cadherin (cs-3195), HDAC6 (cs-7558), phospho-Ser15-p53 (cs-9284), $\gamma \mathrm{H} 2 \mathrm{AX}$ (cs-9718); Abcam: WT1 (ab89901; ab201948); Enzo: HSP90(ADI-SPA-830-F); Millipore: ac-H3 (06-599); Sigma: ac-Tubulin (T7451); BD Biosciences: $\beta$-Catenin (610153), RAC1 (610650); Pharmingen: Poly (ADPRibose) Polymerase (PARP1) (556362). Western blot procedure was done as described (Pons et al., 2018).

\section{Flow cytometry}

For cell cycle analysis, cells were fixed with ice cold $80 \%$ ethanol for $1 \mathrm{~h}$ to 1 week at $-20^{\circ} \mathrm{C}$. After centrifugation, cells were incubated with RNase A (Sigma-Aldrich) in PBS for $1 \mathrm{~h}$ at room temperature and then stained with propidium iodide (SigmaAldrich). Samples were measured and analyzed by flow cytometry in a FACSCanto ${ }^{\mathrm{TM}}$ II as described (Pons et al., 2018).

\section{Proteomics}

Protein expression patterns were determined by global scale proteomics. Sample preparation and mass spectrometry were done as described (Kiweler et al., 2018). In short, proteins were separated on a NOVEX NuPage $10 \%$ gel, proteins were reduced with DTT and subsequently alkylated with iodoacetamide (Sigma). In-gel digestion was done with trypsin (Sigma). Peptides were eluted from the gel with acetonitrile, which was removed in a concentrator (Eppendorf) prior to loading on a C18 StageTip. The measurement was performed on a Q Exactive Plus (Thermo), operated in data-dependent acquisition mode with a top10 method. During the 240 min measurement, peptides were eluted with a $2-40 \%$ acetonitrile/water gradient.
Raw files were processed with MaxQuant (1.5.2.8) (Cox and Mann, 2008) using standard settings, except "match between runs" and "LFQ quantitation" were activated. The search was performed against a human Uniprot database (81194 entries).

LFQ normalized intensities were processed using $\mathrm{R}$ scripts, including filtering of contaminants and putative false positives. For experiments with replicates, imputation for missing values was performed using the bpca method (Stacklies et al., 2007) for proteins detected in at least one replicate. For non-quantified proteins in any replicate, an arbitrary $\log 2$ intensity of 22 was assigned, which is the lowest value across the intensity distribution. Afterwards, mean values were computed for each condition per protein and plotted using the software " $\mathrm{R}$ ".

\section{Transfection}

$1 \times 10^{6} \mathrm{~K} 562$ cells were transfected via electroporation according to the protocol for K562 cells (ATCC ${ }^{\circledR}$ ) with the Ingenio kit solution (Mirus; $100 \mu 1$ solution per sample). $2 \times 10^{6} \mathrm{NB} 4$ cells were electroporated according to the protocol for NB4 cells (ATCC ${ }^{\circledR}$ ) with the Ingenio kit solution (Mirus; $100 \mu 1$ solution per sample) and MV4-11 cells according to the protocol for MV4-11 cells with the Amaxa ${ }^{\circledR}$ Cell Line Nucleofector ${ }^{\circledR}$ Kit V (Lonza). 100 pmol siRNA (nontargeting control siRNA-B/sc-44230 (Santa Cruz) or siRNA targeting HDAC6/LQ-003499-00-0002 (Dharmacon) were electroporated with the Amaxa ${ }^{\circledR}$ Nucleofector ${ }^{\circledR}$ II Device (Lonza) using program T-016 for K562 cells and Q-001 for NB4 and MV4-11 cells. Directly after electroporation, cells were incubated for $24 \mathrm{~h}$ in $2 \mathrm{ml}$ fresh medium per well. Without any washing steps in between, $2 \mathrm{ml}$ fresh medium were added. Cells were harvested after another $24 \mathrm{~h}$.

\section{Alkaline comet assay (also known as single cell gel electrophoresis assay)}

HCT116 cells were seeded at a density of $1 \times 10^{6}$ cells $/ 10 \mathrm{~cm}$ dish. $24 \mathrm{~h}$ later, cells were treated for $24 \mathrm{~h}$ with the topoisomerase-I inhibitor irinotecan or 10-100 $\mu \mathrm{M}$ t-BOOH 20 min before harvesting. The slides were coated with $1.5 \%$ agarose in PBS and dried overnight. $10 \mu \mathrm{l}$ of a cell suspension with $2 \times 10^{6}$ cells $/ \mathrm{ml}$ were diluted in $120 \mu \mathrm{l}$ of $0.5 \%$ in PBS low melting point agarose $\left(37^{\circ} \mathrm{C}\right)$ and added to the coated slides, which were afterwards covered with cover slips and incubated for $5 \mathrm{~min}$ at $4^{\circ} \mathrm{C}$. The cover slips were removed sideward, the slides were incubated for $60 \mathrm{~min}$ in precooled lysis buffer ( $\mathrm{pH}$ 10,2.5 $\mathrm{M} \mathrm{NaCl}, 100 \mathrm{mM}$ EDTA, $10 \mathrm{mM}$ Tris, $1 \%$ sodium lauroylsarcosinate, plus $1 \%$ Triton $\mathrm{X}-100$ and $10 \%$ DMSO added immediately before use) at $4{ }^{\circ} \mathrm{C}$. Slides were placed in an electrophoresis chamber and incubated in precooled electrophoresis buffer $(300 \mathrm{mM} \mathrm{NaOH}, 1 \mathrm{mM}$ EDTA, $\mathrm{pH}>13$ ) at $4^{\circ} \mathrm{C}$ in the dark for 30 min to allow unwinding of the DNA and the exposure of alkali-labile DNA damage. Electrophoresis was performed for $20 \mathrm{~min}$ at $4^{\circ} \mathrm{C}$ at $23 \mathrm{~V}$ and $300 \mathrm{~mA}$ in the dark. Subsequently, the slides were covered 3 times with neutralization buffer $(0.4 \mathrm{M}$ Tris $\mathrm{pH} 7.5)$ for 5 min and briefly immersed in $\mathrm{dH}_{2} \mathrm{O}$. They were then fixed in $100 \%$ ethanol for $5 \mathrm{~min}$. The slides were dried at RT for at least $2 \mathrm{~h}$. DNA was stained with $50 \mu \mathrm{l}$ propidium iodide (stock solution: $50 \mu \mathrm{g} / \mathrm{ml}$, diluted 1:20 in $\mathrm{dH}_{2} \mathrm{O}$ ). The slides were covered with cover slips and directly measured on a fluorescence mi- 
A

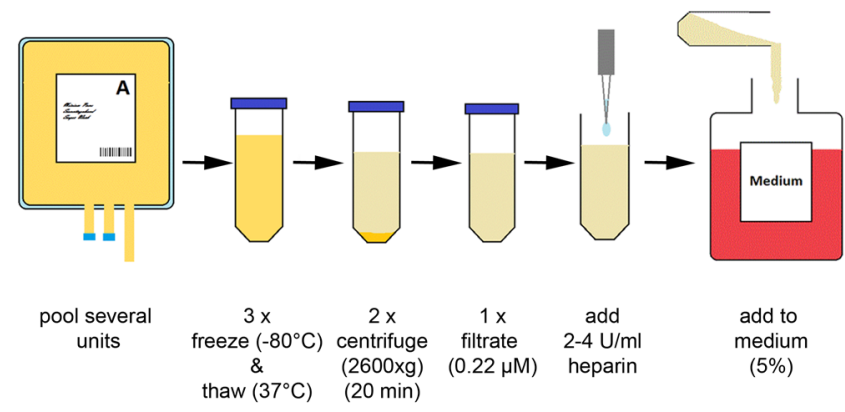

B

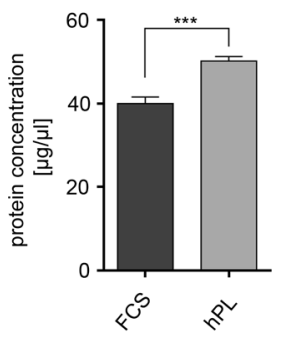

Fig. 1: Process of human platelet lysate production

A) A minimum of 3 units of enriched platelets were pooled. Platelets were frozen at $-80^{\circ} \mathrm{C}$ and thawed at $37^{\circ} \mathrm{C}$ thrice. Two centrifugation steps (2600xg, $20 \mathrm{~min}$ ) removed larger cell debris. The lysate was filtered through a $0.22 \mu \mathrm{M}$ filter to remove smaller debris. To avoid coagulation, $2 \mathrm{U} / \mathrm{ml}$ heparin for suspension cells and $4 \mathrm{U} / \mathrm{ml}$ for adherent cells were added. B) The protein concentrations of three independent stocks of FCS and hPL were compared using the Bradford assay, $n=3$; mean \pm SD; unpaired, two-tailed Student's $t$-test, $\mathrm{p}=0.0008$. croscope (Nikon Instruments). DNA damage (measured as tail intensities) was evaluated with Comet IV software (Perceptive Imaging).

\section{Statistics}

All results were tested for statistical significance with GraphPad Prism 6. When two groups were analyzed, the unpaired, two-tailed Student's $t$-test was used. With more than two groups, a two-way ANOVA test was performed with Bonferroni's multiple comparisons and $95 \%$ confidence interval.

\section{Results}

\subsection{Generation and characterization of hPL}

Figure 1A shows how we generated hPL stocks (Fig. 1A). When we determined the protein concentrations of batches of FCS and hPL, we found that our hPL batch had a slightly higher protein content than the FCS batch (Fig. 1B). The FCS batch that we had chosen was used by us in previous experiments and gave immaculate results regarding pathogen-free cell proliferation (data not shown).

\subsection{Adaptation of cancer cells to media with hPL}

To find out whether leukemic cells (K562, NB4, MV4-11) grow in hPL, we compared the morphology and proliferation of cells that we propagated in RPMI containing $10 \%$ FCS to growth con- ditions in RPMI containing 5\% hPL or 5\% FCS. We performed the same experiment with colorectal cancer cells (HCT116) grown in DMEM with $10 \% \mathrm{FCS}, 5 \% \mathrm{hPL}$, or $5 \% \mathrm{FCS}$.

After 3 weeks of growth in 5\% FCS or $5 \% \mathrm{hPL}$, all four cell lines were morphologically indistinguishable from cells grown in 10\% FCS (Fig. S1 A ${ }^{1}$ ). Furthermore, K562 cells and HCT116 cells grew equally well under the three different conditions (Fig. 2A, $\left.\mathrm{S}^{1} \mathrm{~B}^{1}\right)$. NB4 cells grew best with $10 \% \mathrm{FCS}$ and slightly slower with 5\% FCS and 5\% hPL. MV4-11 cells cultured with 5\% hPL grew as well as those with $10 \%$ FCS, both better than with $5 \%$ FCS (Fig. 2A).

We tested whether cells grown in hPL can be frozen and thawed for continuous propagation. Indeed, cells that had been frozen in medium containing 5\% hPL started to grow similarly to cells that had been frozen with $10 \%$ FCS (Fig. S1B ${ }^{1}$ and data not shown).

Assessment of the cell cycle distributions of leukemic cells and colon cancer cells showed a trend towards fewer dead cells in cultures grown with 5\% hPL (Fig. 2B).

Since HCT116 cells are driven by mutant $\beta$-catenin (Morin et al., 1997), we also assessed $\beta$-catenin expression in HCT116 cells cultured with hPL or FCS. We found no differences in $\beta$-catenin levels under the two culture conditions (Fig. S1C ${ }^{1}$ ). We additionally assessed the levels of the adhesion molecule and epithelial marker protein E-cadherin, which contributes to the regulation of $\beta$-catenin, and of RAC1, which controls epithelial phenotypes (Kimura et al., 2006; Sander and Collard, 1999). These proteins were expressed equally well in HCT116 cells grown with FCS or hPL (Fig. S1C ${ }^{1}$ ).

\footnotetext{
1 doi:10.14573/altex.1809211s
} 
A
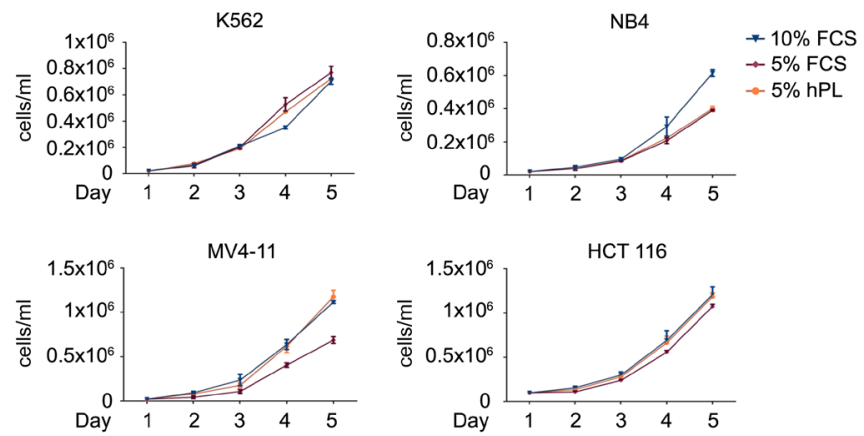

B
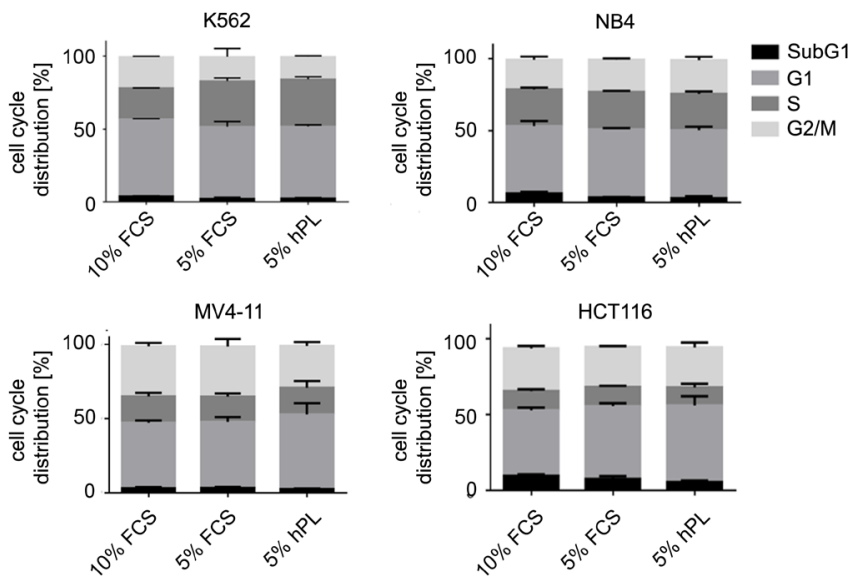

Fig. 2: Cultivation of leukemic and colorectal cells with FCS or hPL A) K562, NB4, MV4-11, and HCT116 cells were adapted to media containing $10 \%$ FCS, $5 \%$ FCS, or 5\% hPL.

Experiments started earliest after 3 weeks adaption time. Growth curves of K562, NB4, MV4-11, and HCT116 cells are shown. Cells were seeded on day 1 at $0.2 \times 10^{4}$ cells $/ \mathrm{ml}$ (K562, NB4, and MV4-11) or $0.1 \times 10^{5}$ cells/well (HCT116) in a 12-well plate and counted after every $24 \mathrm{~h}$ until day 5. ANOVA: K562 grew slower in $10 \%$ FCS than in $5 \%$ FCS $\left({ }^{* * *} p \leq 0.0001\right)$ or $5 \% \mathrm{hPL}\left({ }^{* *} p \leq 0.01\right)$ on day 4 . NB4 cells grew faster in $10 \%$ FCS than in $5 \% \mathrm{FCS}$ or $5 \% \mathrm{hPL}$ (day 3 , ${ }^{*} \mathrm{p} \leq 0.05$; day $\left.4,{ }^{* * *} \mathrm{p} \leq 0.0001\right)$. MV4-11 cells grew slower in 5\% FCS than in 10\% FCS (day $3,{ }^{*} p \leq 0.05$; day $4,{ }^{* * *} p \leq 0.001$; day $5,{ }^{* * *} p \leq 0.0001$ ) and $5 \% \mathrm{hPL}$ (day 4 , ${ }^{* * *} p \leq 0.001$; day $5,{ }^{* * * *} p \leq 0.0001$ ).

HCT116 cells grew slower on day 4 and 5 in $5 \%$ FCS than in $10 \%$ FCS $\left({ }^{* *} p \leq 0.01\right)$ and $5 \% \mathrm{hPL}\left({ }^{*} p \leq 0.05\right)$. B) Cells were analyzed for cell cycle distributions via flow cytometry assessing DNA contents of fixed and PI-stained cells; $n=3$; mean \pm SD; ANOVA: K562 cells have less cells in S-phase when cultured in 10\% FCS than in $5 \% \mathrm{FCS}$ or $5 \% \mathrm{hPL}\left({ }^{* *} \mathrm{p} \leq 0.01\right)$. All other differences did not reach statistical significance.

Fig. 3: Global scale proteomics of K562 cells cultivated with FCS or hPL

A) Three independent replicates of untreated $\mathrm{K} 562$ cells cultured in medium containing $10 \% \mathrm{FCS}$ or $5 \% \mathrm{hPL}$ were analyzed for global protein expression by label-free quantification (LFQ) via mass spectrometry (MS); $\mathrm{n}=3$; mean $\pm \mathrm{SD}$; $1 \%$ false discovery rate (FDR). Heatmap shows changes in LFQ expression levels of proteins. Color gradient represents (log/relative) enrichment of proteins found by MS. B) Correlation plot shows protein abundances (MaxLFQ intensities) from single shot mass spectrometry measurements. All replicate correlation values were greater than 0.98 , and correlation between different culture conditions was at least 0.98 . 
We conclude that cell morphology and cell cycle distribution patterns are comparable during growth in FCS or hPL.

\subsection{Global protein expression of $\mathrm{K} 562$ cells grown in medium with FCS or hPL}

Although we saw no obvious differences between cells cultured in media with $10 \%$ FCS or $5 \% \mathrm{hPL}$, there could still be alterations at the molecular level. To clarify whether such differences exist, we compared triplicates of K562 cells grown with media plus FCS or hPL with an unbiased proteomic assay. We detected 4562 proteins with this approach and the heatmap resulting from these analyses shows very similar protein expression patterns (Fig. 3A). Differences that we noted between the two culture conditions were not significantly higher than differences that we noted among the triplicates. Via a correlation plot, we could show that all samples were at least 98\% identical (Fig. 3B). Not a single protein was expressed differentially under the two culture conditions (Fig. S2 ${ }^{1}$ ).

\subsection{Reactions of CML cells to imatinib}

Since imatinib is a standard drug for the treatment of CML carrying BCR-ABL (t9;21) (Lamontanara et al., 2013), we assessed how

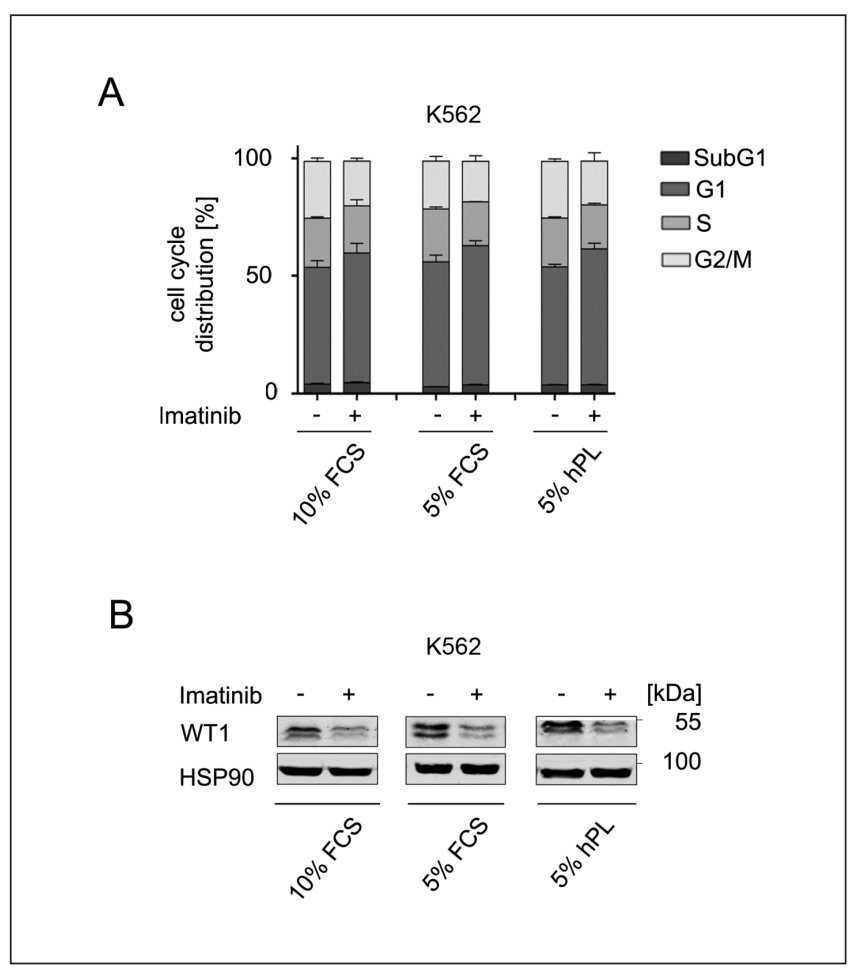

Fig. 4: TKi imatinib equally affects K562 cells cultured with FCS or hPL

A) K562 cells cultivated with $10 \% \mathrm{FCS}, 5 \% \mathrm{FCS}$, or $5 \% \mathrm{hPL}$ were treated with $0.1 \mu \mathrm{M}$ imatinib for $24 \mathrm{~h}$. Cell cycle distributions of $\mathrm{PI}$-stained, fixed cells were analyzed via flow cytometry; $\mathrm{n}=3$; mean \pm SD; ANOVA: Changes caused by imatinib reached statistical significance within each of the three culture conditions ( $\left.{ }^{*} p \leq 0.5\right)$. B) Immunoblot showing expression of WT1 in K562 cells treated as in (a); HSP90, loading control.
BCR-ABL-positive K562 cells cultured in FCS or hPL respond to this drug. Irrespective of culture conditions in 5 or $10 \%$ FCS or $5 \% \mathrm{hPL}$, imatinib reduced the numbers of BCR-ABL-positive $\mathrm{K} 562$ cells in the $\mathrm{S}$ and $\mathrm{G} 2 / \mathrm{M}$ phases and augmented the numbers of cells in G1 phase (Fig. 4A).

Imatinib has been shown to attenuate the expression of WT1 in BCR-ABL-positive leukemic cells (Svensson et al., 2007). We could verify this finding and found an equal reduction of WT1 in $\mathrm{K} 562$ cells that were grown in 5 or $10 \% \mathrm{FCS}$ or $5 \% \mathrm{hPL}$ and imatinib (Fig. 4B).

Hence, culture conditions in FCS and hPL equally allow an assessment of the pharmacological effects of imatinib.

\subsection{Reactions of leukemic cells to HDAC inhibition and regulation of $\mathrm{WT} 1$}

Next, we tested whether cells cultured in media containing hPL or FCS respond differently to pharmacological inhibition of HDACs. HDACi are becoming increasingly appreciated as novel anti-cancer drugs. For our analyses we chose romidepsin, which is approved by the FDA for the treatment of a subset of hematological malignancies (Ceccacci and Minucci, 2016). In addition, we used entinostat and marbostat-100. Like romidepsin, entinostat specifically inhibits class I HDACs HDAC1, -2 , and -3 (Noack et al., 2017). Marbostat-100 is a novel agent that we have recently developed and characterized as the currently most selective inhibitor of HDAC6 (Sellmer et al., 2018; Leonhardt et al., 2018).

We treated K562 cells with romidepsin and marbostat-100 and found that romidepsin led to G1 arrest and a loss of S phase cells in cultures growing with $10 \% \mathrm{FCS}, 5 \% \mathrm{FCS}$, or $5 \% \mathrm{hPL}$ (Fig. 5A). Marbostat-100 did not significantly change cell cycle distribution (Fig. 5A), which is consistent with previous data (Sellmer et al., 2018; Leonhardt et al., 2018).

We found an equal inhibition of HDAC1, -2 and -3 by romidepsin under all three incubation conditions by detection of the surrogate marker acetylated Histone H3 (Krämer et al., 2014) using Western blot. Similarly, we could verify inhibition of HDAC6 by marbostat-100 (Krämer et al., 2014) in FCS- and hPL-based culture conditions by detection of acetylated tubulin using Western blot (Fig. 5B).

It has been found that class I HDACi decrease WT1 mRNA and protein expression (Makki et al., 2008) and it has been speculated that this inhibition may be mediated by inactivation of the chaperone HSP90 (Bansal et al., 2010). Western blot analyses revealed that romidepsin significantly decreased WT1 and caused cleavage of PARP1 in K562 cells cultured in FCS or hPL. Marbostat-100 led to a slight stabilization of WT1 (Fig. 5B,C).

To corroborate that leukemia cells respond equally to structurally diverse HDACi in FCS and hPL, we used MV4-11 cells as an additional system and treated them with entinostat as a further HDACi. PI-staining showed a comparable cell cycle distribution following treatment, independent of culture conditions in FCS or hPL (Fig. S3A $A^{1}$ ). As MV4-11 cells react very sensitively to this agent, we had a closer look at the amount of subG1 fractions, which did also not vary significantly under different culture conditions (Fig. S3B ${ }^{1}$ ). As expected from our data in K562 cells treated with Romidepsin (Fig. 5B), we could observe a loss of WT1 in MV4-11 cells treated with entinostat (data not shown). 
A

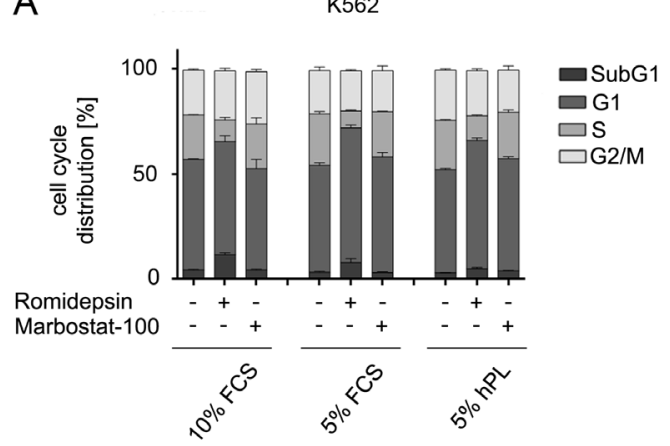

B

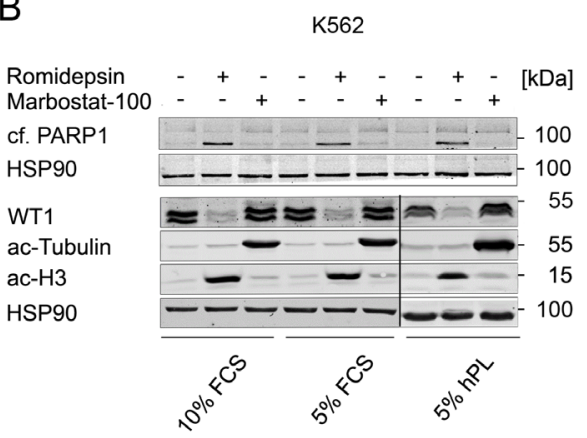

C

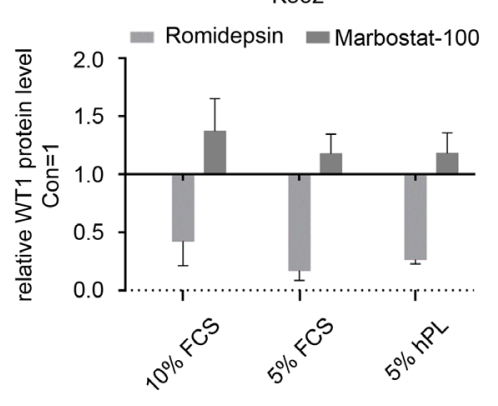

Fig. 5: Influence of HDAC inhibition and knockdown on cell cycle distribution and WT1 protein level in $\mathrm{K} 562$ cells

A) K562 cells were treated with $5 \mathrm{nM}$ romidepsin or $500 \mathrm{nM}$ marbostat-100 for $24 \mathrm{~h}$. For cell cycle distribution analyses, PI-stained, fixed cells were measured by flow cytometry; $n=3$; mean $\pm S D$; ANOVA: Changes caused by romidepsin reached statistical significance within each of the three culture conditions $(p \leq 0.05)$. No significant differences were observed after the treatment with marbostat-100 $(p \geq 0.05)$. B) Levels of PARP1, WT1, ac-tubulin, and ac- $\mathrm{H} 3$ in such cells were analyzed via immunoblot; HSP90, loading control; cf., cleaved form. Since PARP1 was measured with a separate blot, a loading control was also included for this immunoblot. C) Densitometric analysis of WT1 levels after treatment with $5 \mathrm{nM}$ romidepsin or $500 \mathrm{nM}$ marbostat-100 for $24 \mathrm{~h}$. Signal intensities were normalized to the respective loading control. Results display relative amount of $\mathrm{WT} 1 ; n=3$; mean \pm SD; $t$-test: Changes caused by marbostat-100 were not significant $(p \geq 0.05)$ and changes caused by romidepsin reached statistical significance under all culture conditions $\left({ }^{* *} p \leq 0.01\right)$. D) K562 cells cultivated with $10 \%$ FCS or $5 \% \mathrm{hPL}$ were transfected with HDAD6 siRNA via electroporation and incubated for $48 \mathrm{~h}$. Immunoblot shows expression of HDAC6, ac-tubulin, and WT1; HSP90, loading control.
These data illustrate that cells grown in hPL or FCS respond equally to HDACi and that WT1 is attenuated by class I HDACi but not by inhibition of HDAC6.

\subsection{Transfection of cells in media containing hPL or FCS} The genetic manipulation of cultured eukaryotic cells is a mainstay in the search for molecular mechanisms. To assess whether culture conditions based on FCS or hPL have a differential effect on the transfection of leukemic cells with RNAi molecules, we electroporated siRNAs against HDAC6 in K562 (Fig. 5D), MV411, and NB4 cells (Fig. S3C, $D^{1}$ ). We could successfully reduce HDAC6 protein in all three cell lines under both culture conditions (Fig. 5D, S3C, $\mathrm{D}^{1}$ ). As expected from HDAC6 being a tubulin deacetylase (Krämer et al., 2014), the reduction of HDAC6 led to an accumulation of acetylated tubulin (Fig. 5D, S3C,D ${ }^{1}$ ). The levels of WT1 remained constant under such conditions, which supports our data illustrating that a pharmacological inhibition of HDAC6 does not attenuate WT1 expression (Fig. 5B-D).

These data demonstrate that cells grown in hPL or FCS can be transfected with siRNAs and that WT1 is not a target of HDAC6.

\subsection{Responses of leukemic and colon cancer cells to replicative stress}

We next addressed whether leukemic cells and colon cancer cells grown in FCS or hPL respond equally to hydroxyurea. This drug is commonly used to cause replicative stress and to clinically reduce high leukemic blast counts (Eklund et al., 2001; Pons et al., 2018; Schäfer et al., 2017; Schneider et al., 2010; Göder et al., 2018). 
A

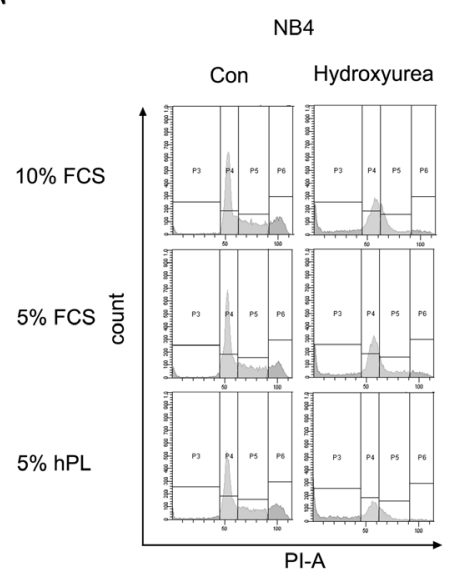

C

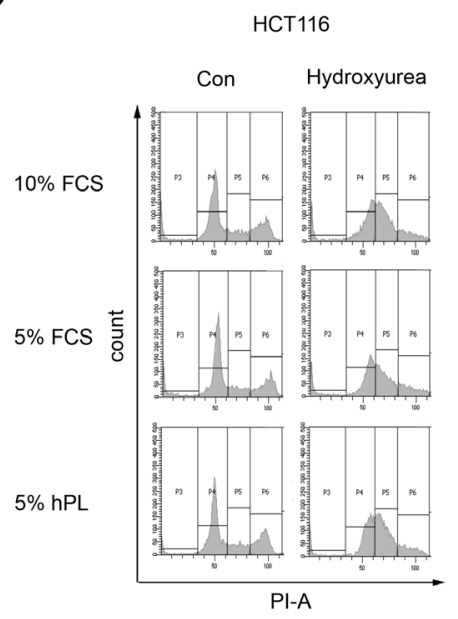

B

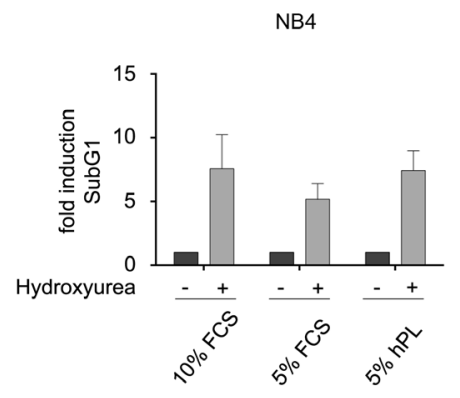

D

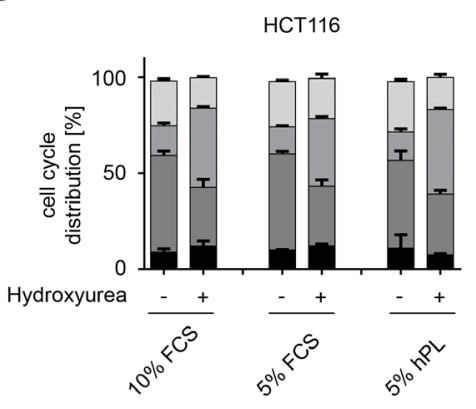

E

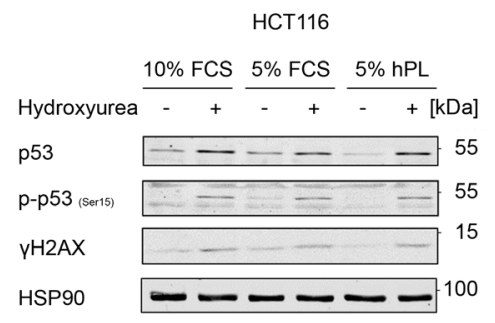

Fig. 6: Induction of replicative stress induced by hydroxyurea reveals no differences in cells grown with FCS or hPL

A) NB4 cells were treated with $0.5 \mathrm{mM}$ hydroxyurea for $24 \mathrm{~h}$. Cell cycle distributions were analyzed via flow cytometry. Representative histograms are shown. B) Induced subG1 levels that were determined by flow cytometry analysis of $\mathrm{PI}$-stained, fixed NB4 cells treated as in (A); $n=3$; mean $\pm \mathrm{SD}$; $t$-test: Changes caused by hydroxyurea reached statistical significance under all culture conditions ( $\left.{ }^{*} p \leq 0.05\right)$. C) HCT116 cells were treated with $1 \mathrm{mM}$ hydroxyurea for $24 \mathrm{~h}$. Representative histograms of cell cycle distributions are shown. D) Cell cycle distributions of HCT116 cells treated as stated in $(C)$ are shown; $n=3$; mean $\pm S D$; ANOVA: Changes caused by hydroxyurea reached statistical significance within the three culture conditions $\left({ }^{\star *} p \leq 0.01\right)$, except for cells cultivated in 5\% FCS, which showed no significant reduction of G2-phase cells ( $p \geq 0.05)$. E) Immunoblot of hydroxyurea-treated HCT116 cells shows expression of p53, p-Ser15-p53, and $\mathrm{YH} 2 \mathrm{AX}$; HSP90, loading control.
Consistent with our previous data (Pons et al., 2018; Göder et al., 2018), hydroxyurea causes replicative stress in HCT116 cells, and NB4 cells rapidly succumb to apoptosis when treated with hydroxyurea. Hydroxyurea induced the number of NB4 cells in subG1, with cells cultured with $10 \%$ FCS and 5\% hPL responding very similarly to hydroxyurea. Cells cultured in 5\% FCS responded slightly less strongly to hydroxyurea, but differences did not reach statistical significance (Fig. 6A,B). In HCT116 cells cultured with $10 \%$ FCS, hydroxyurea induced a 2.7 -fold increase of cells in the S phase. A 2.5-fold increase of cells in S phase occurred in HCT116 cells cultured with 5\% hPL and an 2.9-fold increase of cells in S phase was seen in HCT116 cells cultured with 5\% hPL (Fig. 6C,D).

We have further reported that hydroxyurea could trigger an accumulation of phosphorylated and total forms of the tumor sup- pressor p53 and of the DNA damage marker $\gamma \mathrm{H} 2 \mathrm{AX}$ in HCT116 cells (Göder et al., 2018). HCT116 cells in FCS or hPL equally accumulated these proteins in response to hydroxyurea (Fig. 6E).

We conclude that hydroxyurea evokes similar cellular responses in cancer cells grown in FCS and hPL.

\subsection{Responses of colon cancer cells to irinotecan and $\mathrm{t}-\mathrm{BOOH}$}

Since irinotecan is a standard drug for the treatment of colon cancer (Tomicic and Kaina, 2013), we treated HCT116 cells in medium with 5 or $10 \%$ FCS or $5 \%$ hPL with $5 \mu \mathrm{M}$ irinotecan for $24-48$ $\mathrm{h}$ and analyzed them by flow cytometry for cell cycle distribution and subG1 cells.

After $24 \mathrm{~h}$, irinotecan triggered a strong arrest of cells in the $\mathrm{G} 2 / \mathrm{M}$ phase. This arrest was lost at $48 \mathrm{~h}$ and the portion of cells 


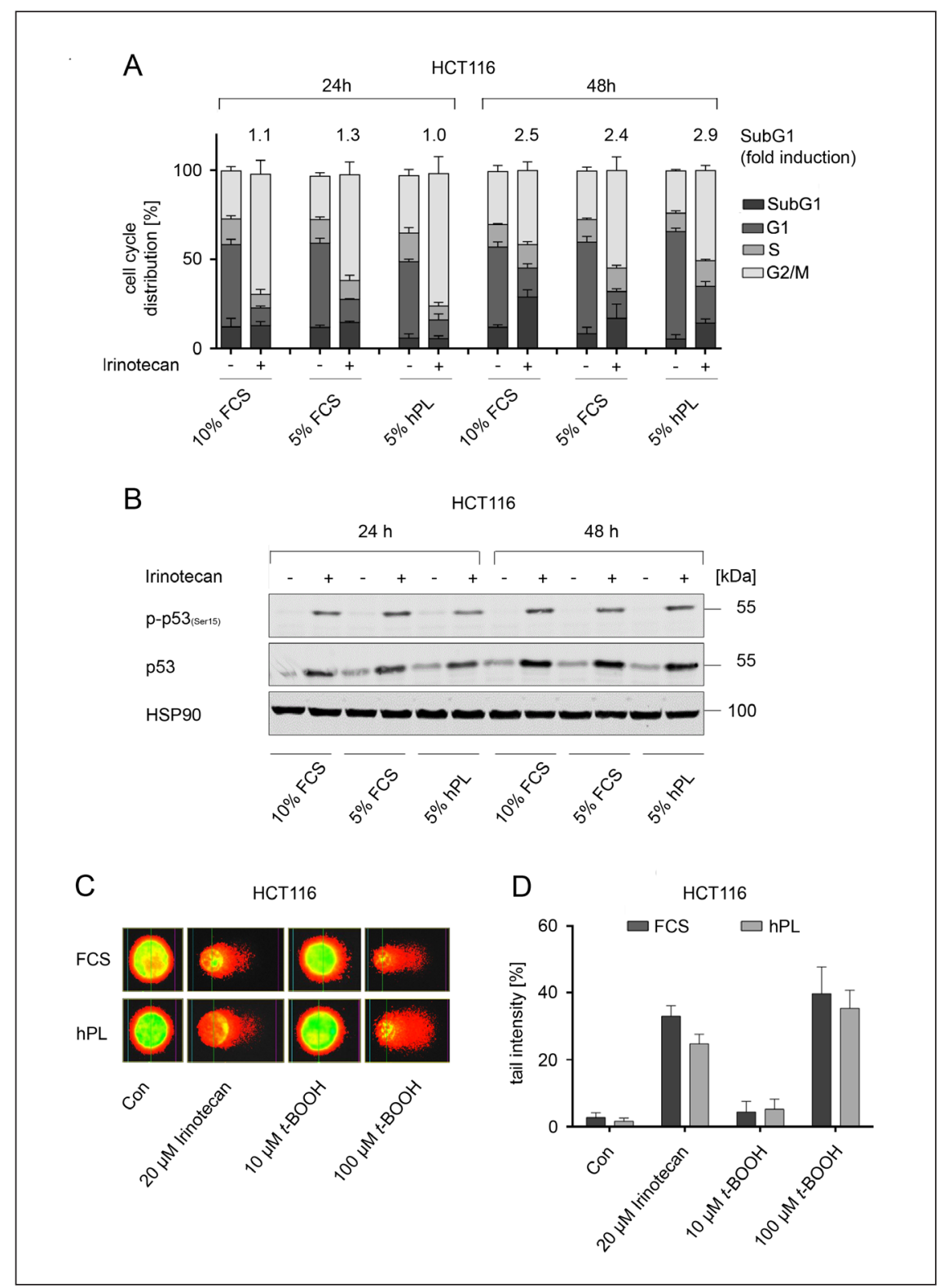

Fig. 7: Irinotecan equally causes DNA strand breaks and activates p53 in HCT116 cells cultivated with FCS and $\mathrm{hPL}$

A) HCT116 cells were treated with $5 \mu \mathrm{M}$ irinotecan for 24-48 h. Cell cycle distributions of PI-stained and fixed cells were determined via flow cytometry; $\mathrm{n}=3$; mean $\pm \mathrm{SD}$; $t$-test: Changes caused by irinotecan reached statistical significance after $24 \mathrm{~h}$ under all culture conditions $\left({ }^{* * * *} p \leq 0.0001\right)$. After $48 \mathrm{~h}$ changes caused by irinotecan reached statistical significance within the three conditions ( $\left.{ }^{* *} p \leq 0.01\right)$, except for cells in the subG1 fraction, which were significantly higher in cells grown with $10 \%$ FCS $\left({ }^{* * *} p \leq 0.0001\right)$. However, the fold induction of cells in subG1 fraction remained nearly the same under all conditions and without significant differences. B) The expression levels of $\mathrm{p} 53$, $\mathrm{p}$-Ser15-p53, and ac-H3 were analyzed by immunoblot; HSP90, loading control. C) Representative pictures of an alkaline comet assay of HCT116 cells cultivated with $10 \%$ FCS or $5 \%$ hPL. Cells were treated with $20 \mu \mathrm{M}$ irinotecan for $24 \mathrm{~h}$ or with $10-100 \mu \mathrm{M} \mathrm{t}-\mathrm{BOOH}$ for 20 min. D) Tail intensities of HCT116 cells that were treated as indicated after alkaline comet assay; $\mathrm{n}=3$; mean $\pm \mathrm{SD}$; $t$-test: No significant differences were observed between FCS and hPL samples ( $p \geq 0.05)$.

in subG1 phase increased (Fig. 7A). Lower levels of subG1 cells were seen in irinotecan-treated HCT116 cells that were grown with $5 \% \mathrm{hPL}$ or $5 \%$ FCS compared to cells that were grown with $10 \%$ FCS. However, this finding should be appreciated with the notion that the subG1 levels are also lower in resting cells that proliferate with 5\% hPL or 5\% FCS (Fig. 7A). Furthermore, the fold induction of the subG1 populations in cells grown with $10 \%$ FCS was 2.5, 2.4 for cells grown with 5\% FCS, and 2.9-fold in cultures grown with $5 \%$ hPL.

Western blot analysis showed that irinotecan caused an about equal accumulation of p-p53 and p53 in HCT116 cells, irrespective of culture conditions in FCS or hPL (Fig. 7B).

Since irinotecan is a DNA-damaging agent, we tested with the single cell electrophoresis assay/comet assay for a potential impact of the two culture conditions on irinotecan-induced DNA damage. In order to analyze ROS-induced DNA damage, we applied t-BOOH (Faust et al., 2017). We exposed cells in 5\% hPL or $10 \% \mathrm{FCS}$ to $20 \mu \mathrm{M}$ irinotecan for $24 \mathrm{~h}$ or to $10 \mu \mathrm{M}$ or $100 \mu \mathrm{M}$ $\mathrm{t}$-BOOH for $20 \mathrm{~min}$. We found that irinotecan as well as t-BOOH evoked equal levels of DNA damage in HCT116 cells grown in FCS or hPL (Fig. 7C,D).

Taken together, these data show that HCT116 cells cultured in hPL or FCS react very similarly to irinotecan.

\section{Discussion}

We report that cells can be adapted to hPL rapidly. Thus, concerns about a time-consuming adaptation of tumor cells to hPL instead of FCS appear unjustified. Data collected with various cell lines 
and types also provide evidence that cells cultured in media containing hPL or FCS respond equally and reproducibly to various types of cellular stress. It is possible that the cultivation of human cells in human lysate is more appropriate than in bovine serum and therefore causes less basal apoptosis and DNA damage. Further experiments will test this hypothesis.

Another concern could be higher costs of hPL over FCS. Depending on the availability of human plasma and possibilities to cooperate with a unit providing (outdated) human plasma, this may or may not be an issue. We would like to stress that the human plasma samples that we used were a few days too old to be given to patients and thus would otherwise be considered medical waste. The feasibility to grow cells in human plasma outdated for clinical use has coherently been reported by others (Rauch et al., 2011; Glovinski et al., 2017). It is surprising that the issue of fresh versus outdated has been raised for hPL when FCS is clearly a material that is stored frozen and upon use is weeks to months old. Furthermore, we find that medium containing 5\% $\mathrm{hPL}$ is equivalent to medium containing $10 \% \mathrm{FCS}$ regarding cell growth and cellular responses to various drugs, which suggests that hPL is twice as potent as FCS. Our finding that the cells we tested grow equally well with $5 \%$ and $10 \%$ FCS further illustrates a worldwide waste of FCS owing to protocols that stipulate the use of $10 \%$ FCS. K562 cells could even grow well with only $1 \%$ FCS (data not shown), which suggests that the use of FCS could be reduced for the cultivation of certain cell types even when one chooses not to substitute FCS for hPL.

Our results that leukemic cells and colon cancer cells grown with FCS or hPL respond comparably to chemotherapeutics that cause replicative stress and DNA damage were anticipated. Comparable responses of leukemic cells to the DNA-damaging agent adriamycin were also seen irrespective of culture in hPL or FCS previously (Fazzina et al., 2016). We additionally assessed cellular reactions to romidepsin, which is one of the most potent specific inhibitors of class I HDACs (Noack et al., 2017). We tested this condition, because endogenous HDACi are present under physiological conditions (Newman and Verdin, 2014; Krämer, 2009). Putative differences in residual endogenous HDACi in FCS or hPL could have altered cellular responses to pharmacological HDACi. However, cells grown with hPL or FCS behaved very similarly when treated with romidepsin.

We further show that inhibition of class I HDACs, but not of HDAC6, leads to a loss of WT1 in K562 cells grown in FCS or hPL. This finding disfavors that a dependency of WT1 stability on HSP90 (Bansal et al., 2010) is linked to HDAC6. Congruent with our data, a lack of interaction between endogenous HDAC6 and overexpressed WT1 has been noted by others in transformed human epithelial kidney cells (Rampal et al., 2014). Hence, HDAC6 seems not to be a component of the HSP90WT1 complex that maintains the stability of WT1. Whether or not HDAC6 deacetylates HSP90 is a matter of debate. HSP90 acetylation and its relevance are more complicated than previously anticipated and deduced from correlations (Krämer et al., 2014). In light of this, marbostat-100 is a precious tool to analyze whether a protein depends on HDAC6. As for WT1, we could rule out a claimed loss of mutant p53 when HDAC6 is inhibited in pancreatic cancer cells (Schäfer et al., 2017; Stojanovic et al., 2017). Furthermore, our observation that inhibitors of class I HDACs reduce WT1 is in agreement with the reduction of WT1 mRNA and an increased proteasomal degradation of WT1 in K562 cells when they are treated with the class I HDACi entinostat (Makki et al., 2008). Like PARP1, WT1 is subject to caspase cleavage (Ruan et al., 2018; Pons et al., 2018). Thus, apoptosis induced by romidepsin is likely linked to the reduction of WT1.

Our findings demonstrate that hPL is a promising replacement for FCS. We will expand our analyses to further cell types and treatment schedules.

\section{References}

Altaie, A., Owston, H. and Jones, E. (2016). Use of platelet lysate for bone regeneration - Are we ready for clinical translation? World J Stem Cells 8, 47-55. doi:10.4252/wjsc.v8.i2.47

Baik, S. Y., Lim, Y. A., Kang, S. J. et al. (2014). Effects of platelet lysate preparations on the proliferation of hacat cells. Ann Lab Med 34, 43-50. doi:10.3343/alm.2014.34.1.43

Bansal, H., Bansal, S., Rao, M. et al. (2010). Heat shock protein 90 regulates the expression of Wilms tumor 1 protein in myeloid leukemias. Blood 116, 4591-4599. doi:10.1182/ blood-2009-10-247239

Barsotti, M. C., Losi, P., Briganti, E. et al. (2013). Effect of platelet lysate on human cells involved in different phases of wound healing. PLoS One 8, e84753. doi:10.1371/journal. pone. 0084753

Bernardi, M., Albiero, E., Alghisi, A. et al. (2013). Production of human platelet lysate by use of ultrasound for ex vivo expansion of human bone marrow-derived mesenchymal stromal cells. Cytotherapy 15, 920-929. doi:10.1016/j.jcyt. 2013.01.219

BMJV - Bundesministerium der Justiz und für Verbraucherschutz (2005). Medicinal Products Act in the version published on 12 December 2005 (Federal Law Gazette [BGBI.]) Part I p. 3394, last amended by Article 3 of the Law of 4 April 2016 (Federal Law Gazette I p. 569).

Bundesärztekammer (2017). Richtlinie zur Gewinnung von Blut und Blutbestandteilen und zur Anwendung von Blutprodukten (Richtlinie Hämotherapie): Gesamtnovelle 2017 - Aufgestellt gemäß $\S 12 \mathrm{a}$ und 18 Transfusionsgesetz von der Bundesärztekammer im Einvernehmen mit dem Paul-Ehrlich-Institut. BAnz AT 06.11.2017 B5. https://www.bundesaerztekam mer.de/fileadmin/user_upload/downloads/pdf-Ordner/MuE/ Richtlinie_Haemotherapie_2017.pdf

Ceccacci, E. and Minucci, S. (2016). Inhibition of histone deacetylases in cancer therapy: Lessons from leukaemia. $\mathrm{Br} J$ Cancer 114, 605-611. doi:10.1038/bjc.2016.36

Cox, J. and Mann, M. (2008). MaxQuant enables high peptide identification rates, individualized p.p.b.-range mass accuracies and proteome-wide protein quantification. Nat Biotechnol 26, 1367-1372. doi:10.1038/nbt.1511

Doucet, C., Ernou, I., Zhang, Y. et al. (2005). Platelet lysates promote mesenchymal stem cell expansion: A safety substitute 
for animal serum in cell-based therapy applications. $J$ Cell Physiol 205, 228-236. doi:10.1002/jcp.20391

Eklund, H., Uhlin, U., Farnegardh, M. et al. (2001). Structure and function of the radical enzyme ribonucleotide reductase. Prog Biophys Mol Biol 77, 177-268. doi:10.1016/S00796107(01)00014-1

Faust, D., Nikolova, T., Watjen, W. et al. (2017). The brassica-derived phytochemical indolo[3,2-b]carbazole protects against oxidative DNA damage by aryl hydrocarbon receptor activation. Arch Toxicol 91, 967-982. doi:10.1007/s00204016-1672-4

Fazzina, R., Iudicone, P., Mariotti, A. et al. (2016). Culture of human cell lines by a pathogen-inactivated human platelet lysate. Cytotechnology 68, 1185-1195. doi:10.1007/s10616015-9878-5

Fernandez-Rebollo, E., Mentrup, B., Ebert, R. et al. (2017). Human platelet lysate versus fetal calf serum: These supplements do not select for different mesenchymal stromal cells. Sci Rep 7, 5132. doi:10.1038/s41598-017-05207-1

Glovinski, P. V., Herly, M., Mathiasen, A. B. et al. (2017). Overcoming the bottleneck of platelet lysate supply in large-scale clinical expansion of adipose-derived stem cells: A comparison of fresh versus three types of platelet lysates from outdated buffy coat-derived platelet concentrates. Cytotherapy 19, 222-234. doi:10.1016/j.jcyt.2016.10.014

Göder, A., Emmerich, C., Nikolova, T. et al. (2018). HDAC1 and HDAC2 integrate checkpoint kinase phosphorylation and cell fate through the phosphatase-2A subunit PR130. Nat Commun 9, 764. doi:10.1038/s41467-018-03096-0

Gstraunthaler, G. (2003). Alternatives to the use of fetal bovine serum: Serum-free cell culture. ALTEX 20, 275-281. http:// www.altex.ch/resources/Altex_2003_4_275_281_Gstraunthaler.pdf

Gstraunthaler, G., Lindl, T. and van der Valk, J. (2014). A severe case of fraudulent blending of fetal bovine serum strengthens the case for serum-free cell and tissue culture applications. Altern Lab Anim 42, 207-209.

Hofseth, L. J., Hussain, S. P. and Harris, C. C. (2004). P53: 25 years after its discovery. Trends Pharmacol Sci 25, 177-181. doi:10.1016/j.tips.2004.02.009

Kimura, K., Kawamoto, K., Teranishi, S. and Nishida, T. (2006). Role of Rac1 in fibronectin-induced adhesion and motility of human corneal epithelial cells. Invest Ophthalmol Vis Sci 47, 4323-4329. doi:10.1167/iovs.05-1508

Kiweler, N., Brill, B., Wirth, M. et al. (2018). The histone deacetylases HDAC1 and HDAC2 are required for the growth and survival of renal carcinoma cells. Arch Toxicol 92, 22272243. doi:10.1007/s00204-018-2229-5

Krämer, O. H. (2009). HDAC2: A critical factor in health and disease. Trends Pharmacol Sci 30, 647-655. doi:10.1016/j. tips.2009.09.007

Krämer, O. H., Mahboobi, S. and Sellmer, A. (2014). Drugging the HDAC6-HSP90 interplay in malignant cells. Trends Pharmacol Sci 35, 501-509. doi:10.1016/j.tips.2014. 08.001

Lamontanara, A. J., Gencer, E. B., Kuzyk, O. and Hantschel,
O. (2013). Mechanisms of resistance to BCR-ABL and other kinase inhibitors. Biochim Biophys Acta 1834, 1449-1459. doi:10.1016/j.bbapap.2012.12.009

Leijs, M. J., van Buul, G. M., Verhaar, J. A. et al. (2017). Pre-treatment of human mesenchymal stem cells with inflammatory factors or hypoxia does not influence migration to osteoarthritic cartilage and synovium. Am J Sports Med 45, 1151-1161. doi:10.1177/0363546516682710

Leonhardt, M., Sellmer, A., Kramer, O. H. et al. (2018). Design and biological evaluation of tetrahydro-beta-carboline derivatives as highly potent histone deacetylase 6 (HDAC6) inhibitors. Eur J Med Chem 152, 329-357. doi:10.1016/j. ejmech.2018.04.046

Lykov, A. P., Bondarenko, N. A., Surovtseva, M. A. et al. (2017). Comparative effects of platelet-rich plasma, platelet lysate, and fetal calf serum on mesenchymal stem cells. Bull Exp Biol Med 163, 757-760. doi:10.1007/s10517-017-3897-5

Makki, M. S., Heinzel, T. and Englert, C. (2008). TSA downregulates Wilms tumor gene 1 (Wt1) expression at multiple levels. Nucleic Acids Res 36, 4067-4078. doi:10.1093/nar/gkn356

Martinelli, D., Pereira, R. C., Mogni, M. et al. (2016). A humanized system to expand in vitro amniotic fluid-derived stem cells intended for clinical application. Cytotherapy 18, 438451. doi:10.1016/j.jcyt.2015.11.020

Moore, P. S., Sipos, B., Orlandini, S. et al. (2001). Genetic profile of 22 pancreatic carcinoma cell lines. Analysis of K-ras, p53, p16 and DPC4/Smad4. Virchows Arch 439, 798-802. doi: $10.1007 / \mathrm{s} 004280100474$

Morin, P. J., Sparks, A. B., Korinek, V. et al. (1997). Activation of beta-catenin-Tcf signaling in colon cancer by mutations in beta-catenin or APC. Science 275, 1787-1790. doi:10.1126/ science. 275.5307 .1787

Nazha, A. and Gerds, A. T. (2016). Where to turn for second-line cytoreduction after hydroxyurea in polycythemia vera? Oncologist 21, 475-480. doi:10.1634/theoncologist.2015-0380

Newman, J. C. and Verdin, E. (2014). Beta-hydroxybutyrate: Much more than a metabolite. Diabetes Res Clin Pract 106, 173-181. doi:10.1016/j.diabres.2014.08.009

Noack, K., Mahendrarajah, N., Hennig, D. et al. (2017). Analysis of the interplay between all-trans retinoic acid and histone deacetylase inhibitors in leukemic cells. Arch Toxicol 91, 2191-2208. doi:10.1007/s00204-016-1878-5

Pons, M., Reichardt, C. M., Hennig, D. et al. (2018). Loss of Wilms tumor 1 protein is a marker for apoptosis in response to replicative stress in leukemic cells. Arch Toxicol 92, 21192135. doi:10.1007/s00204-018-2202-3

Rampal, R., Alkalin, A., Madzo, J. et al. (2014). DNA hydroxymethylation profiling reveals that WT1 mutations result in loss of TET2 function in acute myeloid leukemia. Cell Rep 9, 1841-1855. doi:10.1016/j.celrep.2014.11.004

Rauch, A., Carlstedt, A., Emmerich, C. et al. (2018). Survivin antagonizes chemotherapy-induced cell death of colorectal cancer cells. Oncotarget 9, 27835-27850. doi:10.18632/ oncotarget. 25600

Rauch, C., Feifel, E., Amann, E. M. et al. (2011). Alternatives to the use of fetal bovine serum: Human platelet lysates as a 
serum substitute in cell culture media. ALTEX 28, 305-316. doi:10.14573/altex.2011.4.305

Riis, S., Nielsen, F. M., Pennisi, C. P. et al. (2016). Comparative analysis of media and supplements on initiation and expansion of adipose-derived stem cells. Stem Cells Transl Med 5, 314324. doi:10.5966/sctm.2015-0148

Ruan, J., Gao, S., Yang, J. et al. (2018). WT1 protein is cleaved by caspase-3 in apoptotic leukemic cells. Leuk Lymphoma 59, 162-170. doi:10.1080/10428194.2017.1312368

Sander, E. E. and Collard, J. G. (1999). Rho-like GTPases: Their role in epithelial cell-cell adhesion and invasion. Eur J Cancer 35, 1905-1911. doi:10.1016/S0959-8049(99)00293-2

Schäfer, C., Göder, A., Beyer, M. et al. (2017). Class I histone deacetylases regulate $\mathrm{p} 53 / \mathrm{NF}-\mathrm{kappaB}$ crosstalk in cancer cells. Cell Signal 29, 218-225. doi:10.1016/j.cellsig.2016.11.002

Schneider, G., Henrich, A., Greiner, G. et al. (2010). Cross talk between stimulated NF-kappaB and the tumor suppressor p53. Oncogene 29, 2795-2806. doi:10.1038/onc.2010.46

Sellmer, A., Stangl, H., Beyer, M. et al. (2018). Marbostat-100 defines a new class of potent and selective antiinflammatory and antirheumatic histone deacetylase 6 inhibitors. $J$ Med Chem 61, 3454-3477. doi:10.1021/acs.jmedchem.7b01593

Sergeeva, N. S., Shanskii, Y. D., Sviridova, I. K. et al. (2016). Analysis of reparative activity of platelet lysate: Effect on cell monolayer recovery in vitro and skin wound healing in vivo. Bull Exp Biol Med 162, 138-145. doi:10.1007/s10517-016-3563-3

Shichinohe, H., Kawabori, M., Iijima, H. et al. (2017). Research on advanced intervention using novel bone marrow stem cell (RAINBOW): A study protocol for a phase I, open-label, uncontrolled, dose-response trial of autologous bone marrow stromal cell transplantation in patients with acute ischemic stroke. BMC Neurol 17, 179. doi:10.1186/s12883-017-0955-6

Stacklies, W., Redestig, H., Scholz, M. et al. (2007). pcaMethods - A bioconductor package providing PCA methods for incomplete data. Bioinformatics 23, 1164-1167. doi:10.1093/bio informatics/btm069

Stojanovic, N., Hassan, Z., Wirth, M. et al. (2017). HDAC1 and HDAC2 integrate the expression of p53 mutants in pancreatic cancer. Oncogene 36, 1804-1815. doi:10.1038/onc.2016.344

Svensson, E., Vidovic, K., Lassen, C. et al. (2007). Deregulation of the Wilms' tumour gene 1 protein (WT1) by BCR/ABL1 mediates resistance to imatinib in human leukaemia cells. Leukemia 21, 2485-2494. doi:10.1038/sj.leu.2404924

Tan, C., Shichinohe, H., Wang, Z. et al. (2016). Feasibility and efficiency of human bone marrow stromal cell culture with allogeneic platelet lysate-supplementation for cell therapy against stroke. Stem Cells Int 2016, 6104780. doi:10.1155/ 2016/6104780

Thieme, D., Reuland, L., Lindl, T. et al. (2017). Optimized human platelet lysate as novel basis for a serum-, xeno-, and additive-free corneal endothelial cell and tissue culture. J Tissue Eng Regen Med 12, 557-564. doi:10.1002/term.2574

Tomicic, M. T. and Kaina, B. (2013). Topoisomerase degradation, DSB repair, p53 and IAPs in cancer cell resistance to camptothecinlike topoisomerase I inhibitors. Biochim Biophys Acta 1835, 1127. doi:10.1016/j.bbcan.2012.09.002

\section{Conflict of interest}

OHK, SM, and AS have a patent on marbostat-100 (PCT Int. Appl. (2016), WO 2016020369 A1). RC is working for a manufacturer of apheresis products ( $\mathrm{aPC}$ ).

\section{Acknowledgements}

We thank all members of the Krämer group, Prof. B. Kaina (UM Mainz), and Prof. T. Heinzel (FSU Jena) for helpful discussions. This study was supported by the Deutsche Forschungsgemeinschaft (\#KR2291/5-1 and KR2291/7-1 to OHK) and the Wilhelm Sander-Stiftung (\#2010.078.2 to OHK). 